Helgoländer wiss. Meeresunters. 27, 108-114 (1975)

\title{
Kreuzungsexperimente an Laminaria saccharina von Helgoland und von der Isle of Man
}

\author{
K. LÜNING \\ Biologische Anstalt Helgoland (Meeresstation); \\ Helgoland, Bundesrepublik Deutschland
}

\begin{abstract}
Crossing experiments in Laminaria saccharina from Helgoland and from the Isle of Man. The brown alga Laminaria saccharina growing at Helgoland (North Sea) has smooth blades, whereas the blades of the same species occurring at the Isle of Man are covered with deep bullations. Gametophytes of both morphological types were grown in the laboratory and the resulting sporophytes of each type as well as hybrids were planted out in the sublittoral zone of Helgoland for four months. The Isle of Man type and the hybrids both formed bullations. The Isle of Man type differed further from the Helgoland type in that it had broader blades and did not tolerate the water temperatures above $14^{\circ} \mathrm{C}$ which prevail near Helgoland in summer. All experimental plants were removed from the sea before the blades became sporogenous.
\end{abstract}

\section{EINLEITUNG}

Innerhalb der Gattung Laminaria, die von LAMouroux (1813) aufgestellt wurde, herrscht auch heute noch ein "taxonomisches Chaos" (KAIN, 1971), das bereits von SETCHELL (1893) mit den Worten gekennzeichnet wurde: "The species of Laminaria are at present in great confusion and it is very difficult to determine just how many there are."

Von den über hundert beschriebenen Arten von Laminaria kann man heute nur etwa 30 als gesichert anerkennen, wobei ein Drittel auf die Sektion der Digitatae (Arten mit geschlitztem Phylloid) und zwei Drittel auf die Sektion der Simplices (Arten mit ungeteiltem Phylloid) entfallen (KarN, 1971).

Innerhalb der Sektion der Simplices ist die Abgrenzung von Arten besonders schwierig. Als wichtigste Unterscheidungsmerkmale betrachtete Wrlce (1965) das Vorkommen von Schleimgängen in Phylloid und Stiel sowie den Besitz von massiven oder hohlen Stielen. Inzwischen hat sich herausgestellt, daß gerade diese Merkmale weitgehend durch die Umweltbedingungen bestimmt werden (Burrows, 1964; Chapman, $1973,1974,1975$ ).

Im europäischen Bereich existieren zumindest zwei auffällig verschiedene Formen von Laminaria saccharina (L.) L.AMour. Jedem Beobachter, der die Helgoländer Exemplare von Laminaria saccharina mit Exemplaren von den englischen, französischen und 
westnorwegischen Küsten vergleicht, fällt sofort auf, daß die Phylloide der Helgoländer Pflanzen eine glatte Oberfläche besitzen, während die Phylloide der an den genannten Küsten vorkommenden Exemplare dicht mit Höckern ("Bullae") und Vertiefungen besetzt sind. Von den älteren Autoren hat besonders AGARDH (1867) auf diesen Unterschied hingewiesen. NEWTON (1931) unterschied neben Laminaria saccharina (L.) LAMOUR. (Phylloide glatt oder mit 2 Reihen von nicht stark ausgeprägten Vertiefungen) noch die von AGARDH (1867) aufgestellte L. hieroglyphica (Phylloide mit 4-5 stark ausgeprägten Bullae und alternierenden Vertiefungen), wobei weiter auf einen deutlichen Unterschied in der Form des Sorus hingewiesen wurde. Dieser liegt bei der erstgenannten "Art" als breites, kontinuierliches Band vor, tritt aber bei der zweitgenannten "Art" fleckenhaft auf. Später schlossen PARkE \& Dixon (1968) L. hieroglypbica wieder in Laminaria saccharina (L.) LAMOUR. ein.

Die vorliegende Arbeit beschäftigt sich mit der Frage, inwieweit hier genetisch verschiedene Formen vorliegen, wobei als Vertreter der mit Bullae versehenen Pflanzen Laminaria saccharina von der Isle of Man verwendet und mit dem Helgoländer Material verglichen wurde.

\section{MATERIAL UND METHODEN}

Sporogene Pflanzen von Laminaria saccharina wurden bei Helgoland und bei der Isle of Man (Port Erin) gesammelt. Sorusbedeckte Phylloidpartien wurden trockengeputzt, $12 \mathrm{~h}$ lang bei $10^{\circ} \mathrm{C}$ in geschlossenen Glasschalen aufbewahrt und anschließend mit angereichertem Meerwasser (Provasol., 1966) übergossen. Die ausschlüpfenden Zoosporen bildeten bei $15^{\circ} \mathrm{C}$ in Rotlicht (Leuchtstoffröhren Philips TL $40 \mathrm{~W} / 15$, kombiniert mit 3-mm-Röhm \& Haass-Plexiglas $\mathrm{Nr} .501 ; 2.0 \mathrm{nE} \cdot \mathrm{cm}^{-2} \cdot \mathrm{s}^{-1}$ ) stark verzweigte, vegetativ bleibende Gametophyten (LÜNING \& DRING, 1972). Männliche und weibliche Gametophyten wurden im Alter von 4 Wochen unter dem Mikroskop isoliert und getrennt weitergezüchtet. Im Alter von 3 Monaten wurden die vegetativen Gametophytenbällchen von etwa $1 \mathrm{~mm}$ Durchmesser im Mörser vorsichtig zu Fadenfragmenten zerstoßen. Nach der Vereinigung von männlichen und weiblichen Gametophytenfragmenten wurden diese in angereichertem Meerwasser auf Kunststoffgaze (Maschenweite $1 \times 1 \mathrm{~mm}$ ) bei $10^{\circ} \mathrm{C}$ in Weißlicht (Leuchtstoffröhren Osram $40 \mathrm{~W} / 15 ; 2000 \mathrm{Lux}$ ) weitergezüchtet. Nach wenigen Tagen trat die Fertilisierung ein, und nach 3 Wochen. waren die der Gaze fest ansitzenden Sporophyten etwa $1 \mathrm{~mm}$ lang. Die weitere Anzucht erfolgte in fließendem Meerwasser, worin die Sporophyten nach dreiwöchigem Aufenthalt eine Länge von etwa $1 \mathrm{~cm}$ erreichten. In Parallelkulturen, welche nur weibliche Gametophyten enthielten, hatten sich parthenogenetisch ebenfalls Sporophyten entwickelt, die sich von normalen, diploiden Sporophyten durch unpolares Wachstum und unregelmäßige Zellanordnung unterschieden (SCHreIBER, 1930; ChapMaN, 1974). Die maximal erreichte Thalluslänge der parthenogenetischen Sporophyten war $2 \mathrm{~mm}$.

Vor dem Einbringen der Versuchspflanzen in das Meer wurden Gazestücke $(2 \times 5 \mathrm{~cm})$ mit anhaftenden Sporophyten auf numerierten Kunststoffbrettern $(8 \times 8 \mathrm{~cm})$ mit Hilfe von Kunststoffschrauben montiert. Ende März 1973 wurden die Versuchspflanzen auf Unterwassergitter $(2 \times 1 \mathrm{~m})$ in das Helgoländer Sublitoral $(1 \mathrm{~m}$ 
Wassertiefe) gebracht. Ab Anfang Juni wurden 10 Versuchspflanzen aus jeder Serie in dreiwöchigem Abstand fotografiert und zur Ermittlung der Wachstumsrate in $10 \mathrm{~cm}$ Entfernung vom Stielansatz gelocht. Die Berechnung des linearen Expansionskoeffizienten erfolgte nach Sundene (1964). Ende Juli 1973 wurden alle Versuchsexemplare aus dem Meer genommen und in Alkohol-Glyzerin konserviert.

\section{ERGEBNISSE}

Im Alter von 4 Monaten und nach zehnwöchigem Aufenthalt im Helgoländer Sublitoral wurden jeweils 50-100 der rein- und mischerbigen Versuchspflanzen untersucht (Tab. 1). Alle reinerbigen Versuchspflanzen, welche von Elternpflanzen von der Isle of Man abstammten, hatten auch im Helgoländer Sublitoral Bullae entwickelt, ebenso die reziproken Bastarde $z$ wischen englischen und Helgoländer Pflanzen (Abb. 1), während die reinerbigen Helgoländer Exemplare eine glatte Phylloidoberfläche besaßen.

\section{Tabelle 1}

Vorkommen von Phylloidhöckern (Bullae) bei verschiedenen Kreuzungen von Laminaria saccharina (HEI $=$ Herkunft Helgoland, IOM $=$ Herkunft Isle of Man)

\begin{tabular}{|c|c|c|}
\hline Kreuzungen & $\begin{array}{c}\text { Pflanzen mit Bullae } \\
(0 / 0)\end{array}$ & $\begin{array}{l}\text { Anzahl der untersuchten } \\
\text { Pflanzen }\end{array}$ \\
\hline HEL $Q \times$ HEL $\sigma$ & 0 & 84 \\
\hline IOM $9 \times$ IOM & 100 & 68 \\
\hline HEL $\$ \times$ IOM $\delta$ & 100 & 55 \\
\hline IOM $\$$ HEL $\delta$ & 100 & 99 \\
\hline
\end{tabular}

Allerdings setzt die Ausbildung der Bullae erst ein, wenn die Sporophyten eine Länge von 15-20 cm erreicht haben. Bei $30 \mathrm{~cm}$ langen Phylloiden ist das untere Drittel, bei $40 \mathrm{~cm}$ Länge die untere Hälfte der Phylloide mit Bullae besetzt. Bei älteren und längeren Pflanzen ist das distale, von Bullae freie Phylloidende weitgehend erodiert, so daß diese Pflanzen in der ganzen Länge von Bullae bedeckt erscheinen.

Die Phylloide der reinerbigen Laminaria saccharina von der Isle of Man waren signifikant breiter als die Phylloide der reinerbigen Helgoländer Form und der Bastarde (Tab. 2, Abb. 1). Dagegen waren keine Unterschiede hinsichtlich der Stiellänge festzustellen. Die Wachstumsraten der reinerbigen Pflanzen von der Isle of Man und von Helgoland waren im Juni (Wassertemperatur $11-14^{\circ} \mathrm{C}$ ) etwa gleich, jedoch deutlich höher bei den Bastarden (Tab. 2). Bei den reinerbigen englischen Exemplaren erodierte die distale Phylloidpartie wesentlich rascher als bei der Helgoländer Form und den Bastarden, was in der Phylloidlänge zum Ausdruck kommt (Tab. 2, Abb. 1). Im Juli (Wassertemperatur $14-17^{\circ} \mathrm{C}$ ) verfielen die Phylloide aller reinerbigen, von der Isle of Man stammenden Versuchspflanzen bis auf geringe Reste über dem Stielansatz, und ein Wachstum war nicht mehr festzustellen. Dagegen setzten die reinerbigen Helgoländer Exemplare und die Bastarde auch im Juli ihr Phylloidwachstum fort, wobei die Wachstumsrate der Bastarde gegenüber dem Vormonat um etwa ein Viertel sank. 


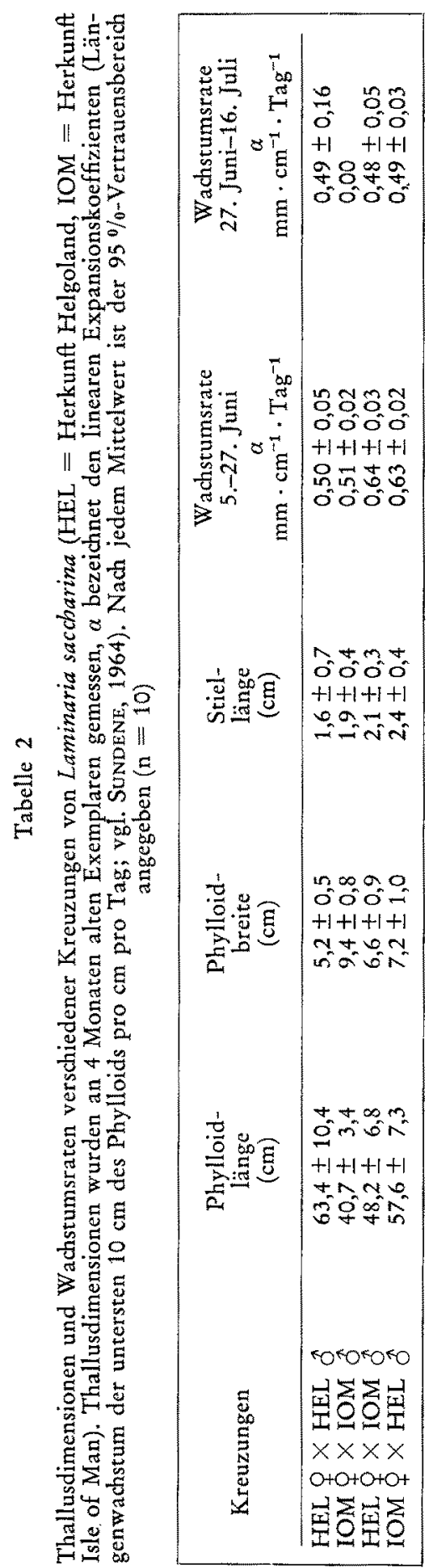


Die mikroskopische Untersuchung ergab, daß sowohl bei den reinerbigen als auch bei den Bastarden Schleimgänge im Phylloid, nicht aber im Stiel vorhanden waren, somit also der Typ (+-) nach WiLcE (1965) vorlag.

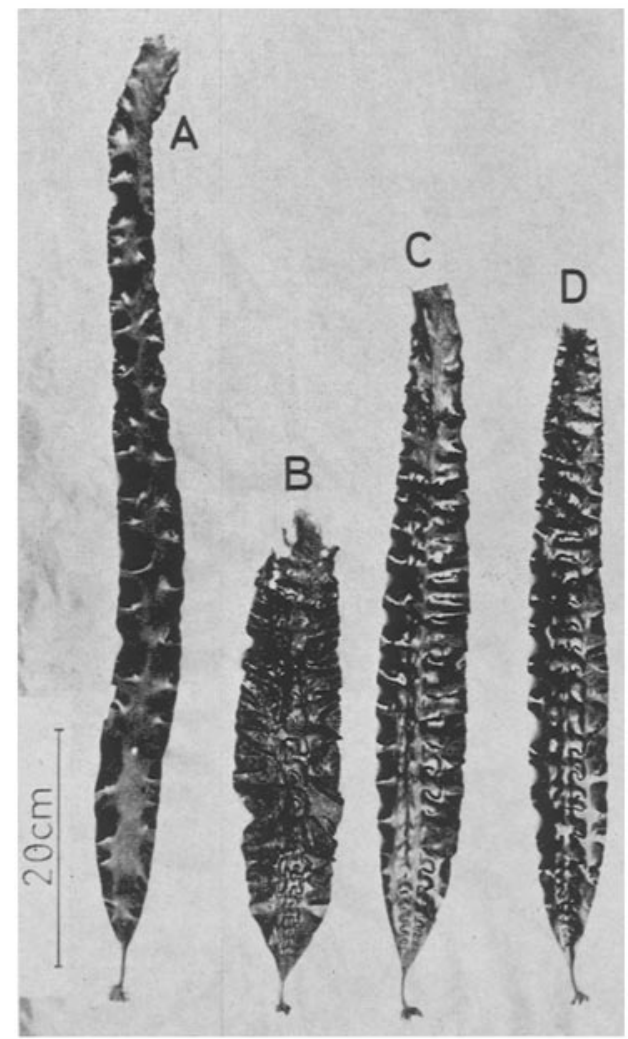

Abb. 1: Laminaria saccharina, bei Helgoland gezüchtet, 4 Monate alt (HEL = Herkunft Helgoland, $\mathrm{IOM}=$ Herkunt Isle of Man). (A) HEL $O \times$ HEL $\delta$, (B) IOM $q \times$ IOM $\delta$, (C) $1 \mathrm{OM} \phi \times \mathrm{HEL} \delta$, (D) HEL $q \times$ IOM $\delta \hat{~}$

\section{DISKUSSION}

Die vorliegende Untersuchung hat gezeigt, daß der auffälligste Unterschied zwischen den englischen und Helgoländer Populationen von Laminaria saccharina, die Ausbildung der Bullae, genetisch bedingt ist. Da beide Gruppen der reziproken Bastarde Bullae besaßen, handelt es sich um ein dominantes, nicht geschlechtsgebundenes Merkmal. Eine Weiterführung des genetischen Experimentes erscheint nicht ratsam, da die Ausbildung des Sorus bei Versuchspflanzen von Laminaria saccharina bisher nicht im Laboratorium erzielt werden kann, im Feld jedoch eine Kontamination der heimischen Flora mit fremden Formen vermieden werden sollte. Die hier durchgeführte viermonatige Exposition der nichtheimischen Versuchsexemplare im Helgoländer Subli- 
toral bedeutete keine Kontaminationsgefahr, da die Pflanzen lange vor der möglichen Ausbildung des Sorus wieder aus dem Meer entnommen wurden und die Möglichkeit einer Refixierung von etwa abgerissenen Versuchspflanzen wegen der starken Strömung im Helgoländer Sublitoral praktisch nicht besteht. Andererseits war die Exposition der Versuchsexemplare im Meer notwendig, da im Laboratorium Laminaria saccharina nur bis zu einer Phylloidlänge von etwa $20 \mathrm{~cm}$ herangezüchtet werden kann und bei solchen Versuchsexemplaren noch nicht alle typischen Merkmale vorliegen.

Weiter wurden Unterschiede in der Phylloidbreite und in der Toleranz gegenüber Wassertemperaturen oberhalb von $14^{\circ} \mathrm{C}$ festgestellt. Diese Wassertemperatur wird an der Isle of Man kaum überschritten, während bei Helgoland im August eine Wassertemperatur von $18^{\circ} \mathrm{C}$ erreicht wird. Möglicherweise tragen die hohen Sommertemperaturen dazu bei, daß die mit Bullae versehene Form von Laminaria saccharina von der Ansiedlung bei Helgoland ausgeschlossen bleibt.

\section{ZUSAMMENFASSUNG}

1. Zwei morphologisch verschiedene Formen der Braunalge Laminaria saccharina (Helgoländer Form mit glatten Phylloiden, Form von der Isle of Man mit Phylloidhöckern) wurden gekreuzt.

2. Die von der Isle of Man stammende Form sowie die reziproken Bastarde entwickelten auch im Helgoländer Sublitoral Phylloidhöcker.

3. Die englische Form hatte breitere Phylloide als die Helgoländer Form und verfiel ab Juli, als die Wassertemperatur bei Helgoland $14^{\circ} \mathrm{C}$ überschritt.

4. Alle Versuchspflanzen wurden nach viermonatigem Aufenthalt wieder aus dem Meer entnommen. Eine Kontamination der Helgoländer Unterwasserflora mit der nichtheimischen Form kann ausgeschlossen werden.

Danksagungen. Für die Ubersendung von sporogenem Material danke ich Frau Dr. J. M. Jones (Department of Marine Biology, Port Erin, Isle of Man). Für wertvolle Hilfe danke ich Frl. Rryelts, Frl. Gronstad, der Tauchgruppe der Biologischen Anstalt Helgoland und der Schiffsbesatzung von MB „Ellenbogen“.

\section{ZITIERTE LITERATUR}

AgardH, J. G., 1867. De Laminarieis symbolas offert J. G. Agardh. Lunds Univ. Ârsskr. 4, $1-36$.

BurRows, E. M., 1964. An experimental assessment of some of the characters used for specific delimitation in the genus Laminaria. J. mar. biol. Ass. U. K. 44, 137-143.

Chapman, A. R. O., 1973. Phenetic variability of stipe morphology in relation to season, exposure and depth in the non-digitate complex of Laminaria Lamour (Phaeophyta, Laminariales) in Nova Scotia. Phycologia 12, 53-57.

- 1974. The genetic basis of morphological differentiation in some Laminaria populations. Mar. Biol. 24, 85-91.

- 1975. Inheritance of mucilage ducts in Laminaria. Proc. int. Seaweed Symp. 8 (in press).

KaIN, J. M., 1971. Synopsis of biological data on Laminaria byperborea. F. A. O. Fish. Synopses 87. 
Lamouroux, J. V. F., 1813. Essai sur les genres de la famille des Thalassiophytes (1) non articulées. Annls Mus. Hist. nat. Marseille 22, 21-47.

Lüning, K. \& Dring, M. J., 1972. Reproduction induced by blue light in female gametophytes of Laminaria saccharina. Planta 104, 252-256.

NewTon, L., 1931. A handbook of the British seaweeds. British Museum, London, 478 pp.

PARKe, M. \& DrXon, P. S., 1968. Check-list of British marine algae - second revision. J. mar. biol. Ass. U. K. 48, 783-832.

Provasoli, L., 1966. Media and prospects for the cultivation of marine algae. In: Cultures and collections of algae. Ed. by A. Watanabe \& A. Hattori. Jap. Soc. Plant Physiol, $1966,63-75$.

SchreIBER, E., 1930. Untersuchungen über Parthenogenesis, Geschlechtsbestimmung und Bastardierungsvermögen bei Laminarien. Planta 12, 331-353.

SETCHELl, W. A., 1893. On the classification and geographical distribution of the Laminariaceae, Trans. Conn. Acad. Arts Sci. 9, 333-375.

Sundene, O., 1964. The ecology of Laminaria digitata in Norway in view of transplant experiments. Nytt. Mag. Bot. 11, 83-107.

WILCE, R. T., 1965. Studies in the genus Laminaria. III. A revision of the North Atlantic species of the simplices section of Laminaria. Bot. gothoburg. 3, 247-256.

Anschrift des Autors: Dr. K. Lüning

Biologische Anstalt Helgoland (Meeresstation)

D-2192 Helgoland

Bundesrepublik Deutschland 